\title{
DIFFUSION OF DYES IN BIOPOLYMERIC HYDROGELS
}

\author{
David VYROUBAL ${ }^{1}$, Martina KLUČÁKOVÁ ${ }^{1}$ \\ ${ }^{1}$ Brno University of Technology, Faculty of Chemistry, Materials Research Centre, Brno, \\ Czech Republic, EU, David.Vyroubal@vut.cz
}

https://doi.org/10.37904/nanocon.2020.3755

\begin{abstract}
This study is focused on diffusion of dyes in biopolymer-based hydrogels. These hydrogels are based on interaction between biopolymer-like electrolytes with oppositely charged surfactants. When polyelectrolytes interact with oppositely charged surfactants, micelle-like nano-containers can be formed. These nanocontainers are able of binding hydrophobic compounds. In this study, combination of modified dextran (diethylaminoethyl dextran) with positive charge and oppositely charged sodium dodecyl sulfate as surfactant was used for preparation of hydrogels. Next type of hydrogel was based on hyaluronan and positive charged surfactant Septonex (carbethoxypendecinium bromide). As a diffusion probes in hydrogels dyes Nile red and ATTO 488 were used. The diffusion of these dyes from aqueous solutions of $\mathrm{NaCl}$ or surfactants into hydrogels was monitored in time. Transport of dyes into structure of hydrogels was characterized by diffusion coefficients and structural parameters of hydrogels.
\end{abstract}

Keywords: Diffusion, hydrogel, polyelectrolyte, dye, surfactant

\section{INTRODUCTION}

Hydrogels are widely used in many disciplines for their unique characteristics. Hydrogels are very similar to biological tissues therefore they are often used for drug delivery systems in medicine $[1,2,3]$. They are biocompatible and biodegradable. Their advantages are reduced side effects, prolonged drug action and low frequency with which drugs need to be administered [4,5]. Because hydrogels contain a large amount of water one of the main problems is that they are not capable of solubilizing hydrophobic compounds. Incompatibility between hydrogels and hydrophobic solutes can be solved by the incorporation of some hydrophobic domains into the hydrogel structure. Hydrophilic network and aqueous internal phase of the hydrogel still provide its biocompatibility while hydrophobic domains enable solubilisation of hydrophobic compounds in the structure of the hydrogel. These hydrogels with the hydrophobic domains can be prepared by the interaction between biopolymer-like electrolytes with oppositely charged surfactants $[6,7,8]$. In this study, cationized dextran and sodium form of hyaluronan were used as polyelectrolytes. Sodium dodecyl sulfate (SDS) and Septonex were used as oppositely charged surfactants. Dextran and hyaluronan are both naturally occurring carbohydratebased biopolymers. Hyaluronan is a linear polysaccharide formed by alternating units of $\beta-1,3$ and $\beta-1,4$-linked $\mathrm{N}$-acetylglucosamine and glucuronic acid [9]. Dextran is bacterial-derived non-linear polysaccharide made up from $\alpha-1,6$-linked D-glucopyranose residues. Appearance and frequency of $\alpha-1,2 ; \alpha-1,3$ and $\alpha-1,4$-linked side chains depends on the production process or the production organism [10]. These biopolymers are non-toxic, biocompatible, high water soluble, biodegradable and offer high content of functional groups usable in crosslinking [11].

In this study, the incorporation of two different dyes into hydrogels based on dextran with SDS and hyaluronan with Septonex was studied. Nile red was chosen as a model of hydrophobic diffusion probe and Atto 488 was chosen as a model of hydrophilic diffusion probe. The aim of this work is to describe absorption capacity and capability of hydrogel to absorb dyes by the diffusion from their solutions into prepared hydrogel. The dyes were used for the incorporation into domains prepared in the hydrogels. Diffusion coefficients and structural 
parameters of these hydrogels were studied to assess the potential for use in the development of drug-carrier systems.

\section{MATERIALS AND METHODS}

In this study, hyaluronan and cationized dextran was used as polyelectrolytes. Diethylaminoethyl-dextran hydrochloride (Sigma-Aldrich, Czech Republic; DEAED) was used in molecular weight $500 \mathrm{kDa}$. Sodium dodecyl sulfate (SDS, $\geq 98,5 \%$ ) was purchased from Sigma Aldrich (Czech Republic) and used as surfactant for dextran. Sodium form of hyaluronan was purchased from Contipro (Czech Republic). Hyaluronan was used in two different molecular weights as $340 \mathrm{kDa}(\mathrm{LMW})$ and $1540 \mathrm{kDa}$ (HMW). Carbethoxypendecinium bromide (Septonex, Czech Pharmacopoeia quality) was obtained from GBNchem Company (Czech Republic) and used as surfactant for hyaluronan.

All stock solutions were prepared in $0.15 \mathrm{M} \mathrm{NaCl}$ solution using purified water (Purelab ELGA system). Salt solution was used because preliminary experiments showed that a non-zero ionic strength on the aqueous medium is important for obtaining gel-like materials [12,13]. Samples of hydrogels was prepared by mixing polyelectrolyte and surfactant stock solutions in a 1:1 volume ratio. The concentrations of initial stock solutions of polyelectrolytes and surfactants are given in Table 1.

Table 1 Concentrations of initial stock solutions used to prepare hydrogels

\begin{tabular}{|c|c|c|c|c|}
\hline Hydrogel & Polyelectrolyte & $\begin{array}{c}\text { Concentration of } \\
\text { polyelectrolyte } \\
(\% \mathbf{w} / \mathbf{v})\end{array}$ & Surfactant & $\begin{array}{c}\text { Concentration of } \\
\text { surfactant (mM) }\end{array}$ \\
\hline D-I & Cationized dextran & 4 & SDS & 400 \\
\hline D-II & Cationized dextran & 4 & SDS & 100 \\
\hline H-I & HMW hyaluronan & 2 & Septonex & 200 \\
\hline H-II & HMW hyaluronan & 2 & Septonex & 100 \\
\hline L-I & LMW hyaluronan & 2 & Septonex & 200 \\
\hline L-II & LMW hyaluronan & 2 & Septonex & 100 \\
\hline
\end{tabular}

Atto 488 and Nile red were both purchased from Sigma Aldrich. Nile red was dissolved in Septonex (200mM for H-I, L-I and 100mM for H-II, L-II) and in SDS (400mM for D-I and 100mM for D-II). Atto 488 was dissolved in Septonex (200mM for H-I, L-I and 100mM for H-II, L-II) or physiological saline $(0.15 \mathrm{M} \mathrm{NaCl})$. The dyes were used in ten different initial concentrations which are given in Table 2. Hydrogels were equilibrated for 24 hours after preparation and separated from the liquid residue. After that hydrogels were covered by $5 \mathrm{~cm}^{3}$ of SDS, Septonex or $\mathrm{NaCl}$ solution with dissolved dye. The concentration decrease of dyes in solution was monitored by means of UV/VIS spectrometry (Hitachi U-3900). The data were used for the determination of dye absorption in hydrogels distribution coefficient and their diffusivity.

Table 2 Initial concentrations of dyes in the solutions of SDS, Septonex and $\mathrm{NaCl}$ used for diffusion experiments

\begin{tabular}{|c|c|c|c|c|c|c|c|c|c|c|}
\hline Solution & $\mathrm{a}$ & $\mathrm{b}$ & $\mathrm{c}$ & $\mathrm{d}$ & $\mathrm{e}$ & $\mathrm{f}$ & $\mathrm{g}$ & $\mathrm{h}$ & $\mathrm{i}$ & $\mathrm{j}$ \\
\hline Dye $(\boldsymbol{\mu M})$ & 0.1 & 0.3 & 0.5 & 0.7 & 0.8 & 0.9 & 1.0 & 3.0 & 5.0 & 7.0 \\
\hline
\end{tabular}

\section{RESULTS AND DISCUSSION}

In this work, several different approaches for the investigation of transport of model dyes into hydrogels were applied. Dyes were prepared in various solutions. Nile red was prepared as its solution in $100 \mathrm{mM}$ and $400 \mathrm{mM}$ SDS for measurements in dextran-based hydrogels and in $100 \mathrm{mM}$ and $200 \mathrm{mM}$ Septonex for hyaluronan- 
based hydrogels. Atto 488 was prepared as its water solution in $0.15 \mathrm{M} \mathrm{NaCl}$ for both types of hydrogels and in $100 \mathrm{mM}$ and $200 \mathrm{mM}$ Septonex for hyaluronan-based hydrogels. Used concentrations of both surfactants SDS and Septonex were much higher than its critical micellar concentration (CMC). Critical micellar concentration of SDS is around $\sim 8 \mathrm{mM}[14,15,16]$ and CMC of Septonex is $\sim 0.8 \mathrm{mM}[15,17,18]$. Therefore, dyes should be completely distributed in the micelles of both surfactants due to concentrations of their solutions high above CMC. On the contrary, Atto 488 was dissolved in 0.15 water solution of $\mathrm{NaCl}$ and therefore we assumed that it diffused in hydrogels in the form of simple (partially dissolved) molecules. The differences between hydrogels based on chemically identical raw materials were probably connected with the use of different concentrations of surfactants on cross-linking, which means different ratios between functional groups in biopolymers and surfactant ions. The theoretical ratio of charges between biopolymer (hyaluronan, cationized dextran) and surfactant (Septonex, SDS) are $~ 1$ for D-II; 2 for H-II and L-II; and 4 for D-I, H-I, LI. We assumed that a part of surfactant can be exhausted for the formation of hydrogel networks. In the case of D-II, H-II and L-II, the large amount of surfactant is consumed for cross-linking of hydrogel and the content of surfactant in pores is low. In contrast for hydrogels D-I, H-I and L-I, this part is relatively small, therefore the pore structure in hydrogel contains solution with surfactant micelles. If we take account of these differences in the dextran-based hydrogels, we can assume the faster diffusion into hydrogel D-II with low theoretical ratio of charges. However, the results of the diffusion coefficients for hyaluronan-based hydrogels are opposite. Diffusion into hyaluronan-based hydrogels with higher theoretical ratio of charges $(\mathrm{H}-\mathrm{I}, \mathrm{L}-\mathrm{I})$ is faster. The probable theory for hyaluronan-based hydrogels is that higher excess of Septonex surfactant non-consumed for cross-linking actively participates in the diffusion and binds dye into its micelles. The examples of the diffusive flux in the dependence on initial dye concentration for dextran-based hydrogels are shown in Figure 1. The rate of diffusion was strongly influenced by initial concentration of dye solution used as the source of diffusion particles. We can see that the diffusive flux is much higher in D-II hydrogels with lower theoretical ratio of charges ( 1). A summary of all diffusion coefficients obtained is then shown in Table 3.
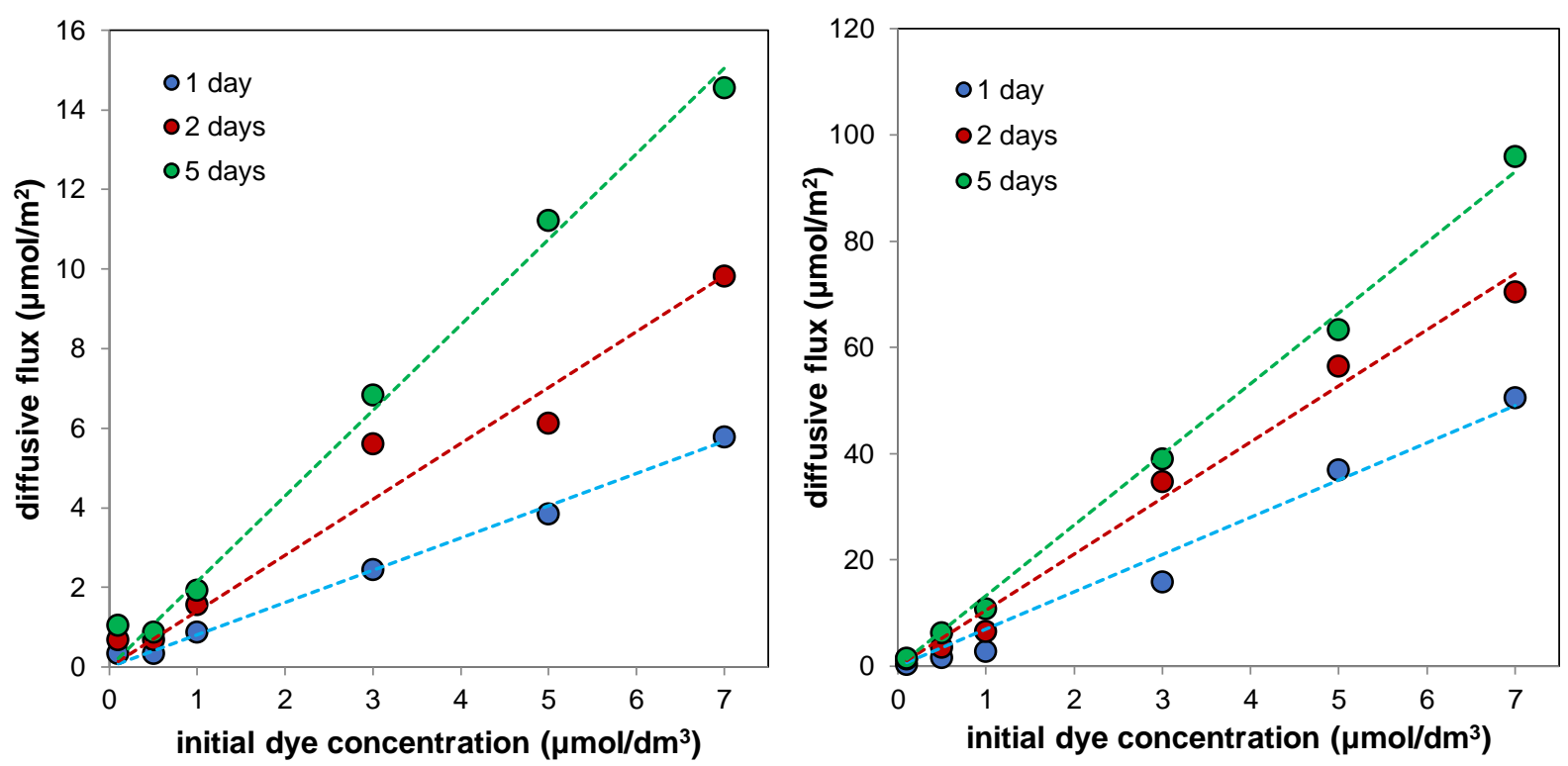

Figure 1 Examples of the diffusive flux in the dependence on initial dye concentration for hydrogels D-I (left) and D-II (right) with Atto 488

The diffusion into hydrogels are more difficult as a result of their intrinstic structure constituted by the hydrogel network and pore structure. Due to various curvatures of pores, the diffusion is strongly influenced not only by the porosity (volume of pores) but also by tortuosity given by their shapes. Hydrogel D-II contains much less free SDS than D-I and its structure should be less cross-linked, therefore the diffusion into the hydrogel D-II is less difficult and the properties of hydrogel has the strong influence. The situation about less or more cross- 
linked hydrogel is the same with hyaluronan-based hydrogels. Hydrogels $\mathrm{H}-\mathrm{I}$ and L-I contains much more Septonex than $\mathrm{H}-\mathrm{II}$ and $\mathrm{L}-\mathrm{II}$ and its structure should be more cross-linked. However the diffusion rate is opposite to dextran-based hydrogels. Which means that the diffusion coefficients are higher in more crosslinked hyaluronan-based hydrogels (H-I, L-I). As already mentioned, this difference is probably due to higher excess of Septonex surfactant non-consumed for cross-linking which actively binds and pulls in dyes from solution into its micelles. Next difference between two studied hydrogels which could affect diffusion coefficients is in the structure of the used biopolymers. Dextran is non-linear bioppolymer with many side chains which depends on production organism and productional process whereas hyaluronan is linear biopolymer. Branching of the dextran chains could affect porosity, volume, size and curvature of the pores and also their tortuozity.

The examples of kinetic data are shown in Figure 2. We can see that the amount of dye diffused into more cross-linked hyaluronan-based hydrogel $\mathrm{H}-\mathrm{I}$ is higher than in less cross-linked $\mathrm{H}$-II. On the other hand, the amount of dye diffused into less cross-linked dextran-based hydrogel D-II is higher than in more cross-linked D-I. The dye content in hydrogels increased strongly mainly in first days.
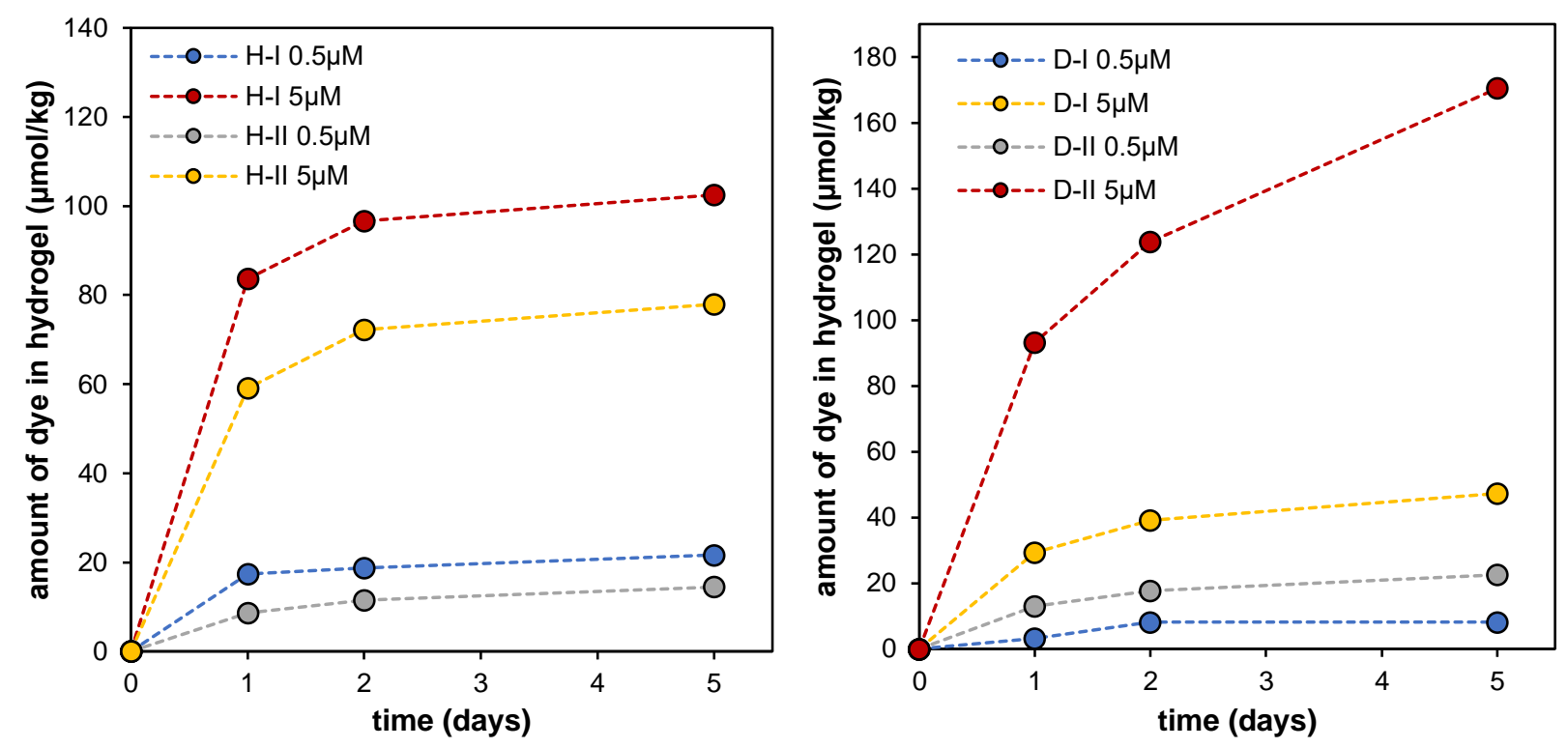

Figure 2 The amount of dyes diffused into hydrogels in the dependence on time: Hyaluronan-based hydrogels with Atto 488 (left) and dextran-based hydrogels with Nile red (right)

Table 3 Effective diffusion coefficients of dyes in hydrogels

\begin{tabular}{|c|c|c|c|c|}
\cline { 2 - 5 } \multicolumn{1}{c|}{} & \multicolumn{4}{|c|}{ Diffusion coefficients $\left(\mathbf{m}^{2} / \mathbf{s}\right)$} \\
\hline Hydrogel & $\begin{array}{c}\text { Atto } 488 \text { in NaCl } \\
\text { solution }\end{array}$ & $\begin{array}{c}\text { Atto } 488 \text { in } \\
\text { Septonex }\end{array}$ & $\begin{array}{c}\text { Nile red in } \\
\text { Septonex }\end{array}$ & Nile red in SDS \\
\hline D-I & $1,55 \times 10^{-10}$ & nd & nd & $4,22 \times 10^{-10}$ \\
\hline D-II & $3,92 \times 10^{-10}$ & nd & nd & $4,63 \times 10^{-10}$ \\
\hline H-I & $1,21 \times 10^{-9}$ & $5,93 \times 10^{-10}$ & $7,86 \times 10^{-10}$ & nd \\
\hline H-II & $4,14 \times 10^{-10}$ & $5,69 \times 10^{-10}$ & $7,40 \times 10^{-10}$ & nd \\
\hline L-I & $7,17 \times 10^{-10}$ & $4,78 \times 10^{-10}$ & $5,53 \times 10^{-10}$ & nd \\
\hline L-II & $5,26 \times 10^{-10}$ & $4,42 \times 10^{-10}$ & $4,28 \times 10^{-10}$ & \\
\hline
\end{tabular}

The values of effective diffusion coefficients listed in Table 3. were obtained on the basis of mathematical model developed by Klučáková et al. [19]. This model was developed for the diffusion couple with phase 
interface. In this work, the couple is comprised by the hydrogel (acceptor part) and the solution of dye above hydrogel (donor part). Both parts are placed in cylindrical vessel. Results published by Zhang et al. showed that the diffusion coefficient of Atto 488 in water is $4.0 \times 10^{-10} \mathrm{~m}^{2} / \mathrm{s}[16,20]$. The diffusion coefficient of Nile red in water is $3.3 \times 10^{-10} \mathrm{~m}^{2} / \mathrm{s}$ [20]. We can see that diffusion coefficients of Atto 488 and Nile red in water are lower (except D-I and D-II hydrogels with Atto 488 in $\mathrm{NaCl}$ solution) in comparison with our obtained results of diffusion coefficients in hydrogels. If we compare our measured diffusion coefficients of dyes in hydrogels with their diffusion coefficients in aqueous solution, then the coefficients in hydrogels have higher values and the diffusion of dyes in hydrogels is faster than in aqueous medium. Thus, higher diffusion coefficients of the dyes in the hydrogels may support the theory that free micelles of surfactants non-consumed for cross-linking located in the pores of hydrogel could accelerate diffusion.

\section{CONCLUSION}

In this work, the diffusion of two different dyes into hydrogels based on a combination of polyelectrolyte and opposite charged surfactant was studied. Hydrogels was based on the combination of cationized dextran with sodium dodecyl sulfate as surfactant and sodium form of hyaluronan with Septonex as surfactant. It was found that the diffusion of dyes into hydrogels is influenced by several factors. One of the factors is amount of surfactant non-consumed for cross-linking located in the pores of hydrogel. Another of the possible factors is difference in branching between linear hyaluronan and non-linear dextran. Diffusion coefficients of Nile red and Atto 488 in the dextran based hydrogel D-II (due to smaller amount of SDS non-consumed for the crosslinking) was much faster than in hydrogel D-I. Diffusion coefficients of Nile red and Atto 488 in hyaluronan based hydrogels $\mathrm{H}-\mathrm{I}$ and $\mathrm{L}-\mathrm{I}$ (which contains higher amount of Septonex non-consumed for the cross-linking) was faster than in hydrogels $\mathrm{H}-\mathrm{II}$ and $\mathrm{L}-\mathrm{II}$.

\section{ACKNOWLEDGEMENTS}

This work was supported by the Czech Science Foundation, project No. 16-12477S and by the project LO1211 from National Programme for Sustainability I (Ministry of Education, Youth and Sports, Czech Republic).

\section{REFERENCES}

[1] LIU, H., WANG, C., LI, C., QIN, Y., WANG, Z., YANG, F., LI, Z. and WANG, J. A functional chitosan-based hydrogel as a wound dressing and drug delivery system in the treatment of wound healing. RSC Advances. 2018, vol. 8, no. 14, pp. 7533-7549.

[2] KONG, B. J., KIM, A., and PARK, S. N. Properties and in vitro drug release of hyaluronic acid-hydroxyethyl cellulose hydrogels for transdermal delivery of isoliquiritigenin. Carbohydrate Polymers. 2016, vol. 147, pp. 473481.

[3] ZHANG, S., ERMANN, J., SUCCI, M. D., ZHOU, A., HAMILTON, M. J., LANGER R. and KARP J. M. An inflammation-targeting hydrogel for local drug delivery in inflammatory bowel disease. Science Translational Medicine. 2015, vol. 7, no. 300.

[4] HUYNH, C. T., NGUYEN, M. K. and LEE, D. S. Injectable block copolymer hydrogels: achievements and future challenges for biomedical applications. Macromolecules. 2011, vol. 44, pp. 6629-6636.

[5] ULLAH F., OTHMAN, M. B. H., JAVED, F., AHMAD, Z. and AKIL, H. M. Classification, processing and application of hydrogels: A review. Materials Science and Engineering: C. 2015, vol. 57, pp. 414-433.

[6] PEKAŘ, M. Hydrogels with micellar hydrophobic (nano)domains. Frontiers in Materials. 2015, vol. 1, no. 35, pp. 1-14.

[7] PICULELL, L. Understanding and exploiting the phase behavior of mixtures of oppositely charged polymers and surfactants in water. Langmuir. 2013, vol. 29, no. 33, pp. 10313-10329. 
[8] LI, D. and WAGNER, N. J. Universal binding behavior for ionic alkyl surfactants with oppositely charged polyelectrolytes. Journal of the American Chemical Society. 2013, vol. 135, no. 46, pp. 17547-17555.

[9] ZHANG, W., JIN, X., LI, H., ZHANG, R. R. and WU, C. W. Injectable and body temperature sensitive hydrogels based on chitosan and hyaluronic acid for $\mathrm{pH}$ sensitive drug release. Carbohydrate Polymers. 2018, vol. 186, pp. 82-90.

[10] MARY HELEN, K.C.B., VETTORIA, P. B., CORTEZIA, M., CRISTIAN J.C. and DE LIMAB, J. B. Dextran: effect of process parameters on production, purification and molecular weight and recent applications. Dialogos \& Ciencia. 2012, vol. 31, pp. 171-186.

[11] OH, J. K., DRUMRUGHT, R., SIEGWART, D. J. and MATYJASZEWSKI, K. The development of microgels /nanogels for drug delivery applications. Progress in Polymer Science. 2008, vol. 33, pp. 448-477.

[12] KROUSKÁ, J., PEKAŘ, M., KLUČÁKOVÁ, M., ŠARAC, B. and BEŠTER-ROGAČ, M. Study of interactions between hyaluronan and cationic surfactants by means of calorimetry, turbidimetry, potentiometry and conductometry. Carbohydrate Polymers. 2017, vol. 157, pp. 1837-1843.

[13] VENEROVÁ, T. and PEKAŘ., M. Rheological properties of gels formed by physical interactions between hyaluronan and cationic surfactants. Carbohydrate Polymers. 2017, vol. 170, pp. 176-181.

[14] UMLONG, I. M. and ISMAIL, K. Micellization behaviour of sodium dodecyl sulfate in different electrolyte media. Colloids and Surfaces, A: Physicochemical Engineering Aspects. 2007, vol. 299, no. 1-3, pp. 8-14.

[15] DUTKIEWICZ, E. and JAKUBOWSKA, A. Effect of electrolytes on the physicochemical behaviour of sodium dodecyl sulphate micelles. Colloid and Polymer Science. 2002, vol. 280, pp. 1009-1014.

[16] ZHANG, X., PONIEWIERSKI, A., JELINSKA, A., ZAGOZDZON, A., WISNIEWSKA, A., HOU, S. and HOLYST, R. Determination of equilibrium and rate constants for complex formation by fluorescence correlation spectroscopy supplemented by dynamic light scattering and Taylor dispersion analysis. Soft Matter. 2016, vol. 12, no. 39, pp. 8186-8194.

[17] KARGEROVÁ, A. and PEKAŘ, M. Ultrasonic study of hyaluronan interactions with Septonex - a pharmaceutical cationic surfactant. Carbohydrate Polymers. 2019, vol. 204, pp. 17-23.

[18] FISCHER, J. and JANDERA, P. Chromatographic behaviour in reversed-phase high-performance liquid chromatography with micellar and submicellar mobile phases effects of the organic modifier. Journal of Chromatography B. 1996, vol. 681, pp. 3-19.

[19] KLUČÁKOVÁ, M. and PEKAŘ, M. Transport of copper(II) ions in humic gel - new results from diffusion couple. Colloids and Surfaces, A: Physicochemical and Engineering Aspects. 2007, vol. 349, no. 1-3, pp. 96-101.

[20] ZHANG, L. X., CAO, X. H., CAI, W. P. and LI, Y. Q. Observations of the effect of confined space on fluorescence and diffusion properties of molecules in single conical nanopore channels. Journal of Fluorescence. 2011, vol. 21, no. 5 , pp. $1865-1870$. 